\title{
Expressing the expected product images in product design of micro-electronic products
}

\author{
Ming-Chuen Chuang ${ }^{\mathrm{a}, *}$, Yung-Chuan $\mathrm{Ma}^{\mathrm{b}}$ \\ a Institute of Applied Arts, National Chiao Tung University, 1001 Ta-Hsieh Rd., Hsinchu, Taiwan, Republic of China \\ ${ }^{\mathrm{b}}$ Industrial Design Division, Acer Incorporate, 21Fl., 88 Sec. 1, Hsin Tai Wu Rd., Hsichih, Taiwan, Republic of China
}

Received 2 January 1999; accepted 30 June 1999

\begin{abstract}
In order to explore how to effectively express product image when designing products, micro-electronic products were used as examples in this study to conduct a comprehensive investigation. Firstly, through a questionnaire survey, we deduced that the five most expected images perceived in micro-electronic products are: (i) high-technology, (ii) efficiency, (iii) lightness and handiness, (iv) nobility, and (v) delicacy. Next, sample product photographs were presented to the subjects in order to make various evaluations. Through semantic differential analysis, factor analysis and multidimensional scaling analysis, the subjects' perception of these images for the evaluated products was revealed and a perceptual map of these products derived. Design subjects' perception was compared to that of non-design subjects. Finally, through morphological analysis of these products, the critical 'form elements' and approaches of form treatment among these products were extracted. By using multiple linear regression, the relationships between each image and various form features were concluded. These findings can be further applied not only to establish a desired image in designing products, but also to reduce the perceptual gap between designers and users.
\end{abstract}

\section{Relevance to industry}

In the development of micro-electronic products, methods enabling the designer to create an appropriate image for a product so that it may communicate with the user are always critical issues. From this study, the expected images of micro-electronic products and the corresponding form features to achieve these images were extracted. A design guideline was summarized accordingly by which the time and cost of product development can be reduced while the quality of the product designed can be enhanced. (C) 2001 Elsevier Science B.V. All rights reserved.

Keywords: Product image; Form elements; Form treatment; Micro-electronic products; Multidimensional scaling

\section{Introduction}

In purchasing and using products, customers or users generally expect to perceive some critical

\footnotetext{
*Corresponding author. Fax: +886-3-5712-332.

E-mail address: ming@iaa.nctu.edu.tw (M.-C. Chuang).
}

images or indications of quality from the product/s. Designers are supposed to provide these images in the products they design. In the past, designers have always assumed that they know what images will be expected and therefore designed products intuitively. However, do designers really know what images users want? Even if they know exactly 
what images to express, do the users perceive the same expected images the designers have aimed to express in products? Are there specific 'form features' or 'treatments' which if adopted or avoided can promote the communication of expected images?

All of the above issues are critical and must be seriously considered when designing products especially due to the continuous minimization and de-materialization of micro-electronic components used in today's products. "Form follows function" is no longer the rule in developing the shape of a product (Fischer, 1989). Instead, designers have to create the form for the product thereby expressing the specific characteristic and image of the product. In this context, the above issues have become more and more important. However, systematic and scientific research on product image or product style is comparatively sparse.

Amongst the few researchers concerned with this subject, Mastandrea et al. (1992) have investigated the communication process between designers and users and the relationship between product images and form elements. The relationship between product images and form elements concluded in that study is not clear. On the other hand, Takahashi (1995) has found a correspondence between some abstract pictorial expressions and the psychological feelings to be communicated.

Dating from the $1970 \mathrm{~s}$ to the present, the socalled "Kansei (Sensational) Engineering" has been continually developed in Japan led by Nagamachi (Nagamachi, 1995). In this field of study, various survey techniques and statistical analyses, such as semantic difference survey (Osgood et al., 1957), morphological analysis, factor analysis, multiple linear regression, neural network simulation and so on have been applied to establish the correspondence between image database and color or design database. Consequently, a computer supported design system can be constructed based on this correspondence. So far, "Kansei Engineering" has been successfully implemented in various product designs, for example in a car, an office chair (Jindo et al., 1995), a pair of spectacles (Chuang and Shih, 1996) and an earth-moving machine (Nakada, 1997).
Recently, the multidimensional scaling (MDS) method, a method to recovering the perceptual space of stimuli from the subjects' judgements of similarity among stimuli (Green et al., 1989), has been applied to investigate users' perception of product forms. For example, Caplan and Faulkner (1982) have implemented MDS to study the perception and preference of users on the configuration of a disk camera for the Kodak Company. By using MDS, Maurer et al. (1992) conducted a study on the semantics of street furniture; whereas Espe (1992) has studied the symbolic quality of watches.

In the study of design style, Chan (1992, 1993) has pioneered a series of quantitative researches on individual style in architecture. Following almost the same idea of Chan (1994) and Chuang and Shiau (1998) investigated the critical factors constituting the style of products in which Ming Dynasty style chairs were used as examples. In that study, an MDS analysis was also performed to explore the perceptual structure of people in distinguishing the form of Ming Dynasty style chairs.

In the present study, ideas and techniques conducted by the researchers mentioned above were applied in order to investigate the expected images and their correspondence to the critical form features in micro-electronic products. Five aspects were studied: (i) the expected images in micro-electronic products, (ii) the representative products to these expected images, (iii) the psychological factors under these expected images, (iv) the perceptual space of micro-electronic products, and (v) the correspondence between the expected images and critical form features in micro-electronic products.

\section{Method}

To explore the five issues, several surveys as described in the following sections were conducted.

\subsection{Survey on expected images}

To investigate the micro-electronic products' expected images, a list of 60 image words deemed 
suitable for expressing the product image was initially screened out by five experienced Industrial Designers. By using this list as a reference, 68 subjects (37 males, 31 females of which 27 were design educated subjects and 41 were non-design educated subjects,) were then asked to write down approximately 10 'image' words that summed up their perceptions of micro-electronic products. Among the 27 design educated subjects, 17 subjects majored in Industrial Design, the other 10 subjects majored in other design fields. Ninetyone percent of the subjects were aged from 20 to 40 years of age, which is purported to be the potential micro-electronic product consumer age group. In comparing the frequency of each image word selected, the 5 most expected images of microelectronic products were decided upon for the following studies. Also, from this the expected images amongst the subjects from different backgrounds can be analyzed.

\subsection{Survey on the representative products to the expected images}

In this survey, 75 products, including 50 microelectronic products, were screened out from various international contemporary design reference books (Sokolov, 1990; Viemeister, 1993; Pearlman, 1996; Industrial Forum Design Hanover, 1996, 1997; Japanese Association of Industrial Design Promotion, 1997) as a 'reference pool'. These products were randomly presented as mounted $3^{\prime \prime} \times 5^{\prime \prime}$ color photos to 87 subjects, including 53 males, 34 females of which 41 were design educated subjects and 46 were non-design educated subjects. The subjects were asked to pick out three of the 75 products that best expressed the image for each of the five expected images. The subjects were also encouraged to explain the reasons for their selections.

\subsection{SD survey on expected images}

An SD (semantic differential) test with 14 opposite adjective pairs to investigate the meanings of the five expected images and 30 product pictures was then conducted over 31 subjects. The 30 products evaluated here comprised 16 repre- sentative products to expected images concluded from the previous survey and 14 products picked randomly from the reference product pool. The 14 opposite adjective pairs were selected to include the advice of some experienced designers. A factor analysis of the collected data was performed to derive the structure of mental feeling in perceiving the expected images and product forms.

\subsection{Survey on the similarity of form among micro-electronic products}

To investigate the subjects' perceptual structure of these products, 35 subjects, including 19 males and 16 females, were asked to sort the above 50 micro-electronic product pictures in the reference product pool into 5-10 piles, according to the similarity of form among the products. An MDS analysis was then conducted to convert this similarity data into a perceptual space representing how subjects perceive these products and expected images.

\subsection{Morphological survey on the product form}

Finally, a morphological analysis of the 30 products, evaluated in the SD survey, was conducted by Industrial Design specialists. This analysis included two steps: firstly, three Industrial Designers, each with 5 years experience, were asked to identify the products' critical form features. Secondly, three more Industrial Designers, each with 8 years experience, were then asked to evaluate whether each of the identified form features appeared in each of the products or not. The result of this analysis was analyzed by a multiple linear regression to deduct the correspondence between expected images and form features or treatments in micro-electronic products.

\section{Results and discussions}

Various analyses were conducted on the data collected from the above surveys to derive results for each of the five aspects in this study. These results will be described and discussed in the following sections, respectively. 
Table 1

The top 10 expected images of microelectronic products

\begin{tabular}{llll}
\hline Rank & Image & Frequency & Percentage \\
\hline 1 & High-tech & 57 & 84 \\
2 & Efficiency & 48 & 71 \\
3 & Lightness and handiness & 42 & 62 \\
4 & Nobility & 38 & 59 \\
5 & Delicacy & 33 & 49 \\
6 & Fashion & 32 & 47 \\
7 & Durability & 28 & 41 \\
8,9 & Convenience, novelty & 26 & 38 \\
10 & Safety & 24 & 35 \\
\hline
\end{tabular}

\subsection{Expected images of micro-electronic products}

The top 10 expected (most frequently selected) images of micro-electronic products are summarized in Table 1. It shows that generally a 'hightech' image is the most expected one. The following expected images can be listed in the order: efficiency, lightness and handiness, nobility, delicacy, fashion, durability, convenience, novelty value and safety.

The expected images of the male subjects and those of the female ones did not vary too greatly. However, the male subjects concentrated mainly on fewer images whilst that of the female subjects spread over more images. Design educated subjects placed more emphasis on images of product form, such as fashion, nobility, delicacy, lightness and handiness (usefulness), sleek, and novelty value images than non-design educated subjects. However, the non-design educated subjects were also concerned with images related to product use, such as images of durability and convenience, in addition to the above images of product form. Furthermore, subjects with an industrial design background showed additional expectations regarding images of safety and convenience perceived in micro-electronic products. Despite the slight difference of expected images amongst the subjects from differing backgrounds, the first five expected images were almost always selected by more than half of the subjects. Thus, these five expected images could therefore be explored further in the following analyses.

\subsection{Products representing the expected images}

By counting the frequency of each product picked out to represent each of the five expected images, the three most frequently selected products for each image were determined and are shown in Fig. 1. The open numbers under the products denote the selection order by the design educated subjects; whereas the closed numbers denote the selection order by the non-design educated subjects. There are several overlaps between their selections. Furthermore, several products have been simultaneously selected to represent different expected images. Consequently, there are 16 products, not 30 products, which appear in Fig. 1. By examining these products, a preliminary conclusion may be proposed - that products representing 'high-tech' or 'efficient' images usually follow a geometrical form; whilst products with 'light' (i.e. portable) and 'handy' image are always small, compact and thin; and products with a 'delicate' or 'noble' image often receive a special surface material finishing treatment.

The frequency of each product picked to represent an expected image was considered as the expressive power of this product to represent the specific image. This index will be used in further analyses.

\subsection{Psychological factors under the expected images}

The data collected from the SD survey was processed by factor analysis. The result, as shown in Table 2, reveals that the 14 opposite adjective pairs used to configure the structure of feeling under the expected images and product form can be classified into three main factors with $82.6 \%$ of variance explained. The first factor, which accounts for $53.2 \%$ of the variance, includes the adjective pairs: beautiful-ugly, delicate-wild, softhard, distinct-negligible, warm-cold, vivid-dull, and light-heavy. This factor may be concluded as one of emotion. The second factor, which accounts for $17 \%$ of the variance and includes the adjective pairs: dignified-frivolous, stable-unstable, rationalemotional, angular-smooth, may be interpreted as that of stability. The third factor, accounting for 


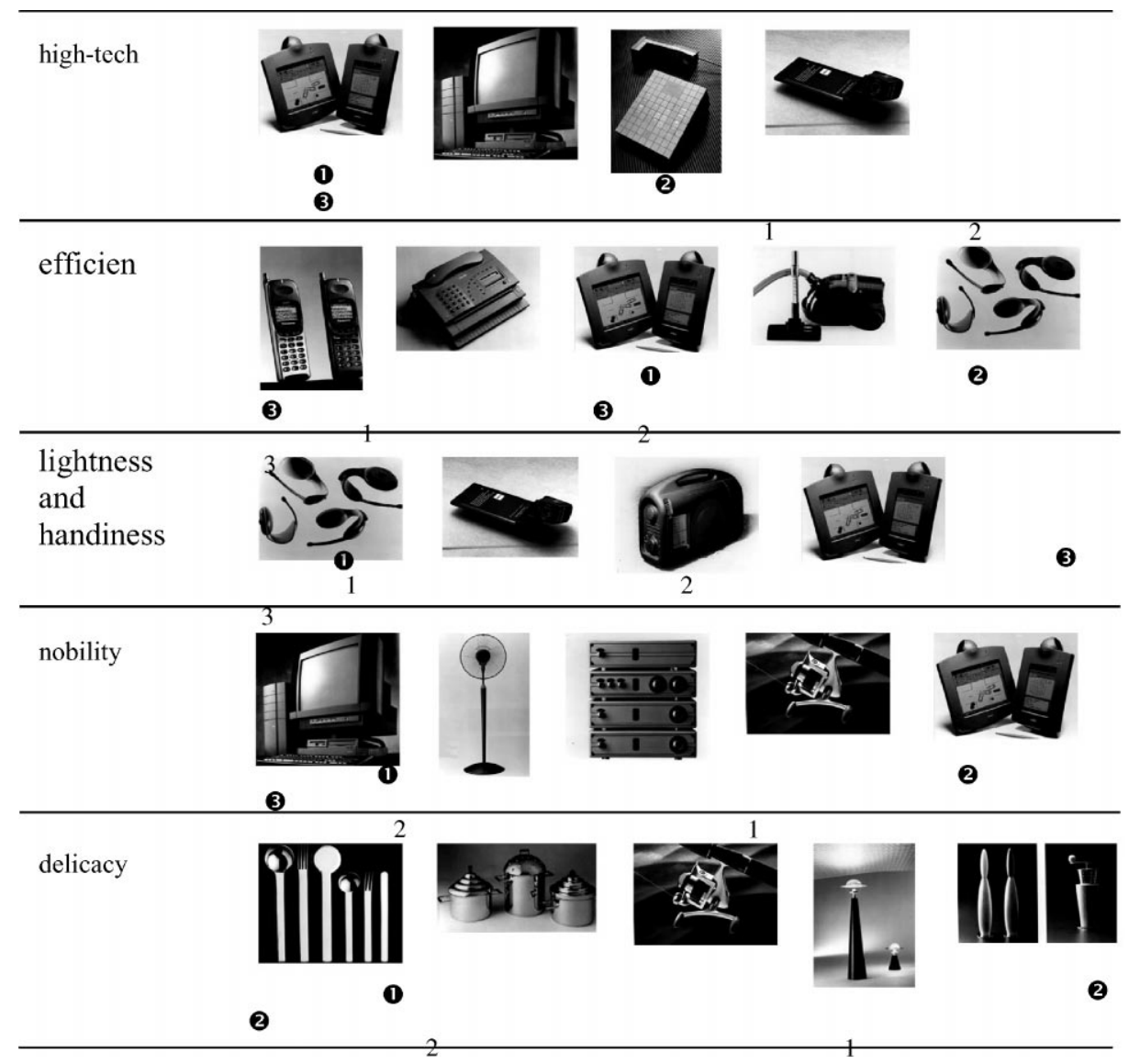

Fig. 1. The representative products to the five most expected images selected by subjects (open numbers denote the selection order by the design subjects; closed numbers denote the selection order by the non-design subjects).

$12.3 \%$ of the variance, includes the adjective pairs: complex-simple, luxurious-plain and unique-general may be interpreted as the factor of simplicity.

These three factors (emotion, stability, simplicity) can be adopted to construct a 'mental space' in the interpretation of individuals' feeling under the expected images. The factor scores of the five most expected images in these three factors can then be treated as the coordinates in this space. Fig. 2 depicts the factor scores and the locations of the five most expected images in this space with 2 two-dimensional plots. It can be deduced from these plots that the image of 'high-tech', 'nobility' and 'delicacy' are similar, because all of them are located in the first quadrants on both plots (all of the coordinates are positive). In particular, the points of 'nobility' and 'delicacy' of image are very close to each other. These two images are almost identical. In general, these three images are supported by a sensational, rational, stable, but luxurious feeling. However, the score in factor 2 of the 'high-tech' image is comparatively higher denoting the more rational and stable feeling of this image whereas the 'noble' and 'delicate' images have relatively higher scores in factor 1 to reveal the more sensational feeling of these images. The factor scores of the 'efficient' image are similar to those of the 'high-tech' image, except the negative score in factor 3. Thus, the image corresponds to a 'sensational', 'rational', 'stable', 
Table 2

The factor loadings of the 14 adjective pairs

\begin{tabular}{lcrr}
\hline Adjective & Factor 1 & Factor 2 & Factor 3 \\
\hline Beautiful-ugly & $\mathbf{0 . 8 9 7 3 2}$ & 0.17404 & 0.14658 \\
Delicate-wild & $\mathbf{0 . 8 6 8 1 3}$ & -0.00930 & 0.24987 \\
Soft-hard & $\mathbf{0 . 7 6 0 6 7}$ & 0.53191 & -0.08350 \\
Distinct-negligible & $\mathbf{0 . 7 2 3 7 1}$ & 0.02443 & 0.56895 \\
Warm-cold & $\mathbf{0 . 7 0 8 6 9}$ & 0.59336 & 0.04635 \\
Vivid-dull & $\mathbf{0 . 6 7 2 5 9}$ & 0.67177 & 0.07768 \\
Light-heavy & $\mathbf{0 . 6 5 2 7 6}$ & 0.56348 & -0.03966 \\
Dignified-frivolous & -0.05868 & $\mathbf{0 . 9 4 8 0 6}$ & -0.00679 \\
Stable-unstable & 0.08836 & $\mathbf{0 . 9 0 4 9 0}$ & -0.25996 \\
Rational-emotional & -0.36028 & $\mathbf{0 . 8 1 5 6 6}$ & 0.00181 \\
Angular-smooth & -0.57485 & $\mathbf{0 . 6 8 8 5 4}$ & -0.01107 \\
Complex-simple & -0.35108 & -0.04713 & $\mathbf{0 . 8 2 5 9 1}$ \\
Gorgeous-plain & 0.34921 & 0.18370 & $\mathbf{0 . 7 9 9 3 3}$ \\
Unique-general & 0.40729 & 0.07247 & $\mathbf{0 . 6 9 9 3 7}$ \\
Eigenvalue & 7.450 & 2.382 & 1.726 \\
Percentage & 53.2 & 17.0 & 12.3 \\
Cumulative percentage & $\mathbf{5 3 . 2}$ & $\mathbf{7 0 . 2}$ & $\mathbf{8 2 . 6}$ \\
\hline
\end{tabular}
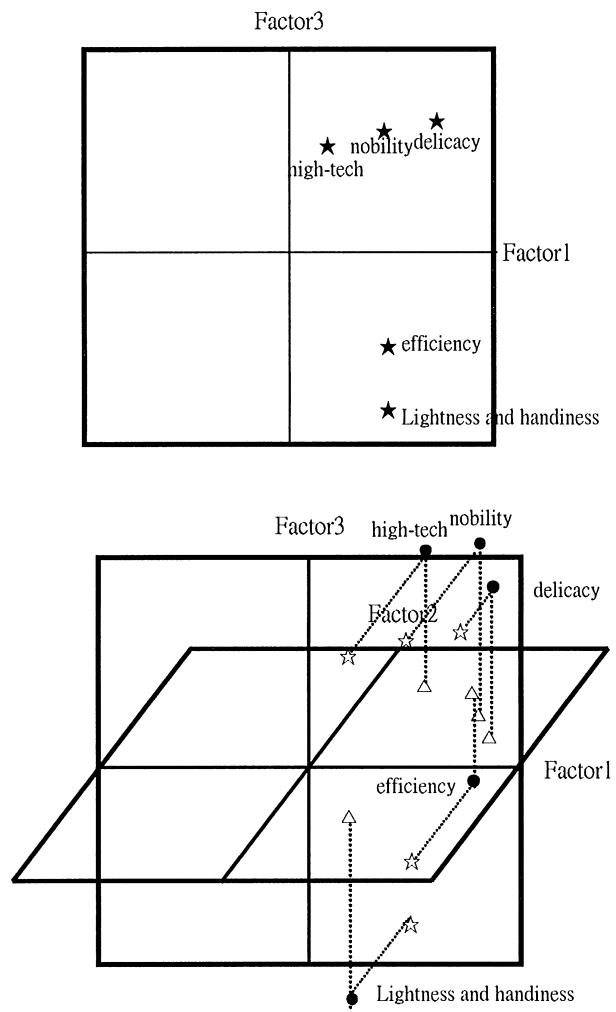

'simple', and 'plain' feeling. The 'light and handy' image has negative scores in both factors 2 and 3, particularly in factor 3 . Thus, this image is very close to the 'simple' and 'plain' feeling and is somewhat related to a 'frivolous' and 'emotional' feeling.

The factor scores of the 30 products evaluated in the SD survey can also be calculated and treated as coordinates in this space. By mapping these 30 points of products into this space, the distances between each product and each expected image were computed. It is reasonable to define the distance as the degree of expressive power of a product to an expected image; the shorter the distance is, the more expressive the product is of this image. Therefore for each expected image, we have two sets of indices denoting the expressive power of products: one is the frequency of direct selection as depicted in the last section, the other is

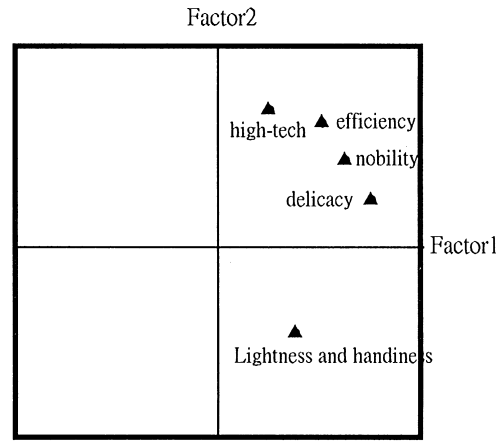

\begin{tabular}{crrr}
\hline \multirow{2}{*}{ image } & \multicolumn{3}{c}{ factor score } \\
\cline { 2 - 4 } & \multicolumn{1}{c}{1} & \multicolumn{1}{c}{2} & \multicolumn{1}{c}{3} \\
\hline high-tech & .66 & 1.61 & 1.62 \\
Efficiency & 1.40 & 1.64 & -1.73 \\
lightness and handiness & 1.33 & -.93 & -2.51 \\
nobility & 1.52 & 1.22 & 1.83 \\
delicacy & 2.05 & .77 & 1.88 \\
\hline
\end{tabular}

Fig. 2. The locations of the five most expected images in the space derived from factor analysis. 

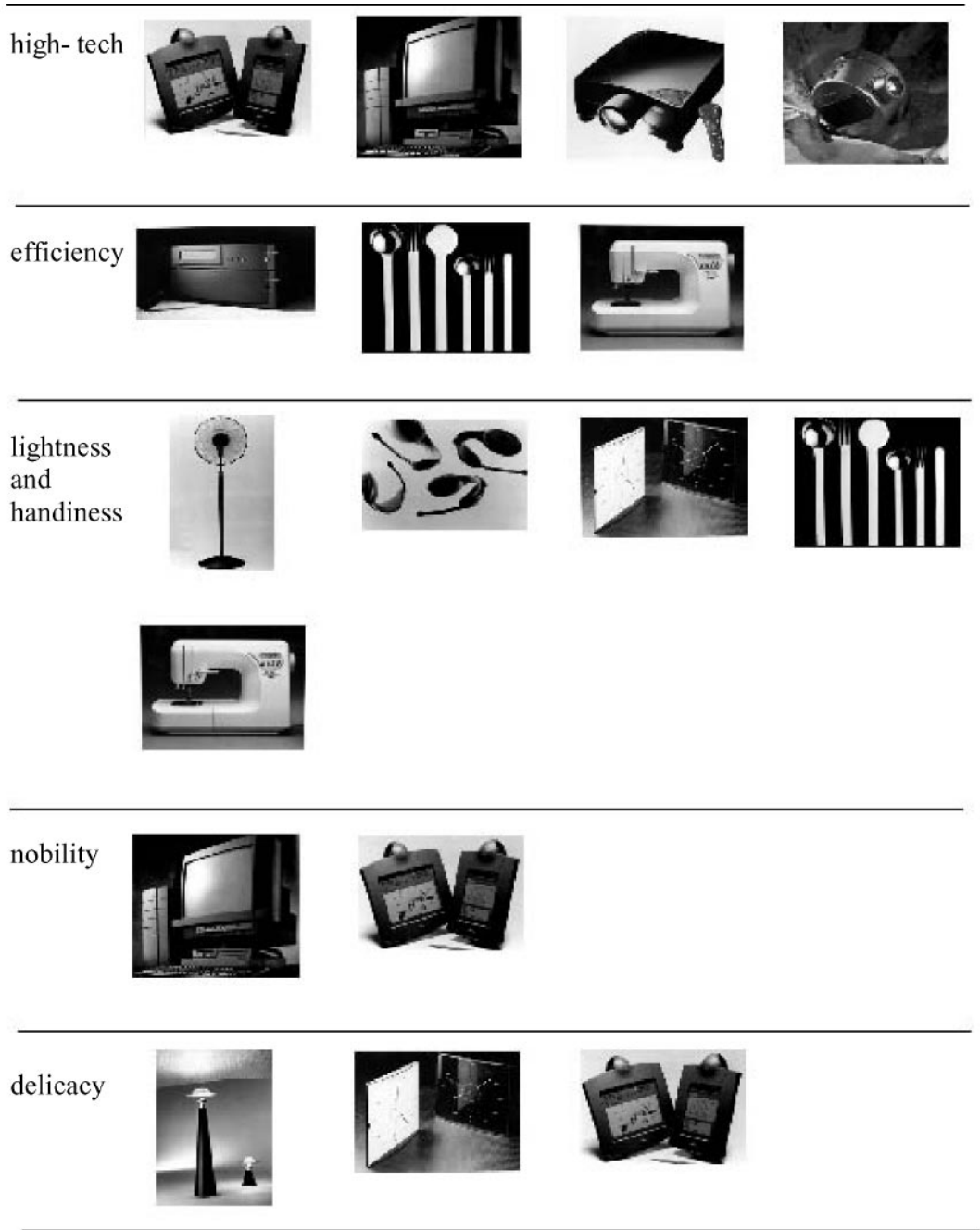

Fig. 3. The representative products to the five most expected images, summarized from direct and indirect measures.

the indirect measurement of distances. The agreement between these two sets of result is fair. Fig. 3 summarizes some products that are representatives of the expected images according to both measures.

\subsection{Perceptual space of micro-electronic products}

The frequency of each of the product pairs which have been grouped into the same pile by subjects in the similarity evaluation were initially summarized into a similarity matrix among these
50 micro-electronic products. This matrix was then analyzed by an MDS program, ALSCAL.

It resulted in a fairly acceptable $\left(R^{2}=0.64\right)$ perceptual space of two dimensions through an appropriate rotation, as shown in Fig. 4 (the $R^{2}$ is 0.71 for a three-dimensional solution.) From this perceptual map, we can find that the product forms located on the upper side are more complex than those on the lower side; while the product forms located on the right side are lighter than those on the left side. This observation is further verified with the high correlation between $X$ 


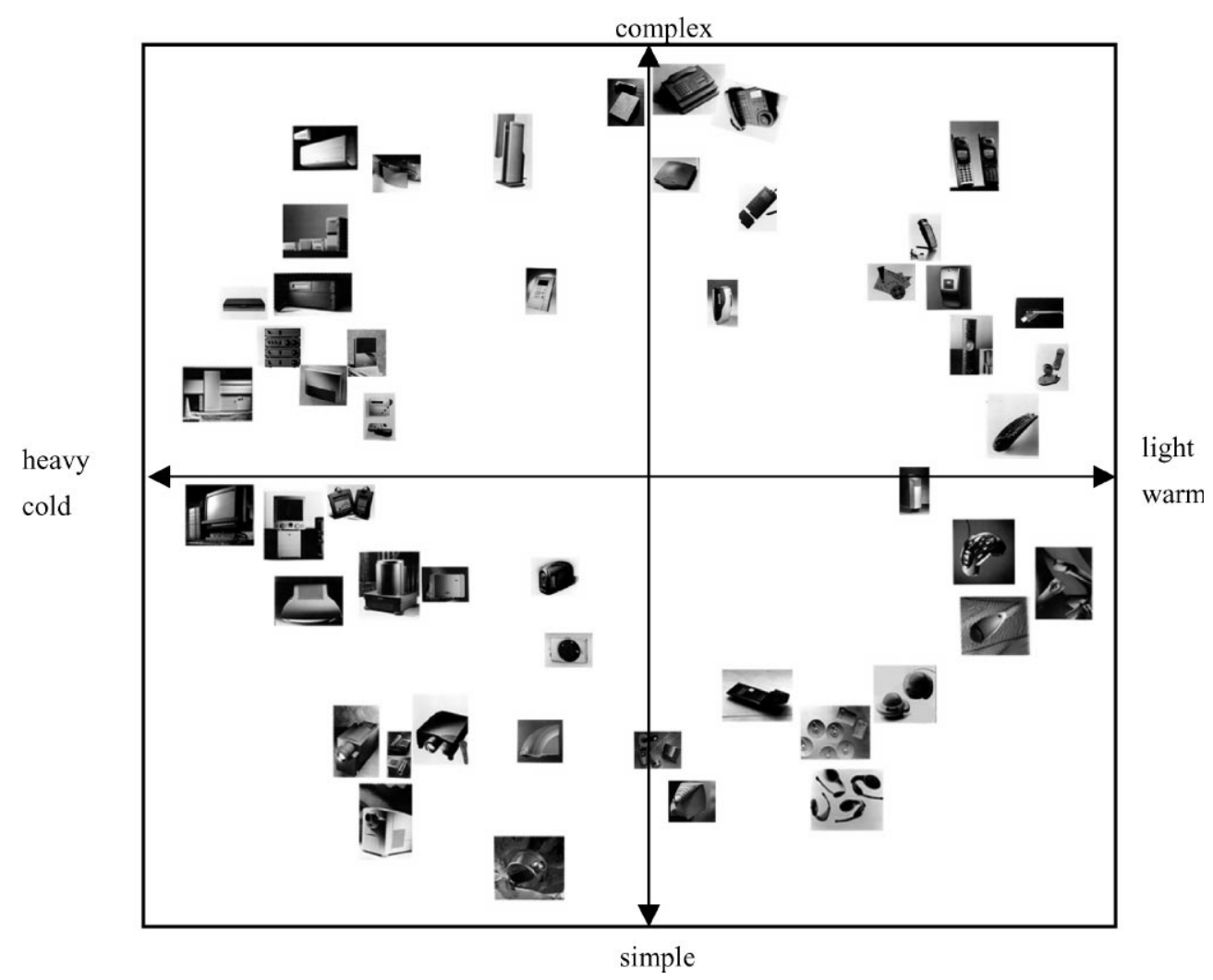

Fig. 4. The perceptual space of micro-electronic products derived from an ALSCAL analysis.

coordinates of products and their corresponding light-heavy scores; and between $Y$ coordinates of products and their corresponding complex-simple scores in the previous SD test. The $X$ coordinates of products are also found to be highly correlated to their corresponding warm-cold scores. Thus, we may conclude that our differentiation between the forms of micro-electronic products is based on our perception on the two major factors: the degree of 'lightness' and 'warmth' of the form as well as the degree of 'form simplicity'.

We then treated the expressive power of the product to the expected images as the degree of preference for this product by the 'image' subject. Through a PREFMAP analysis on this set of data, based on the perceptual map, the ideal points for the 5 expected images could be mapped on the perceptual plane of products, as shown in Fig. 5.
In this figure, ideal points of 'high-tech', 'light and handy', 'noble', 'delicate' images are displayed as solid points to represent positive ideal points; the closer to this ideal point a product is located, the more representative of this image the product is. The ideal point of the 'efficient' image, on the other hand, is a negative ideal point shown as an empty point; the closer to this ideal point a product is located, the less efficiency the product expresses. Both ideal points of a 'noble' and 'hightech' image are located on the upper-left of this map. Thus, products with somewhat complex and heavy forms are more likely to express these images. The ideal point of a 'delicate' image is located near the original point of this map. This means that products with a moderate form, not too light, heavy; complex or monotonous, will be perceived as a 'delicate' image. Because the ideal 


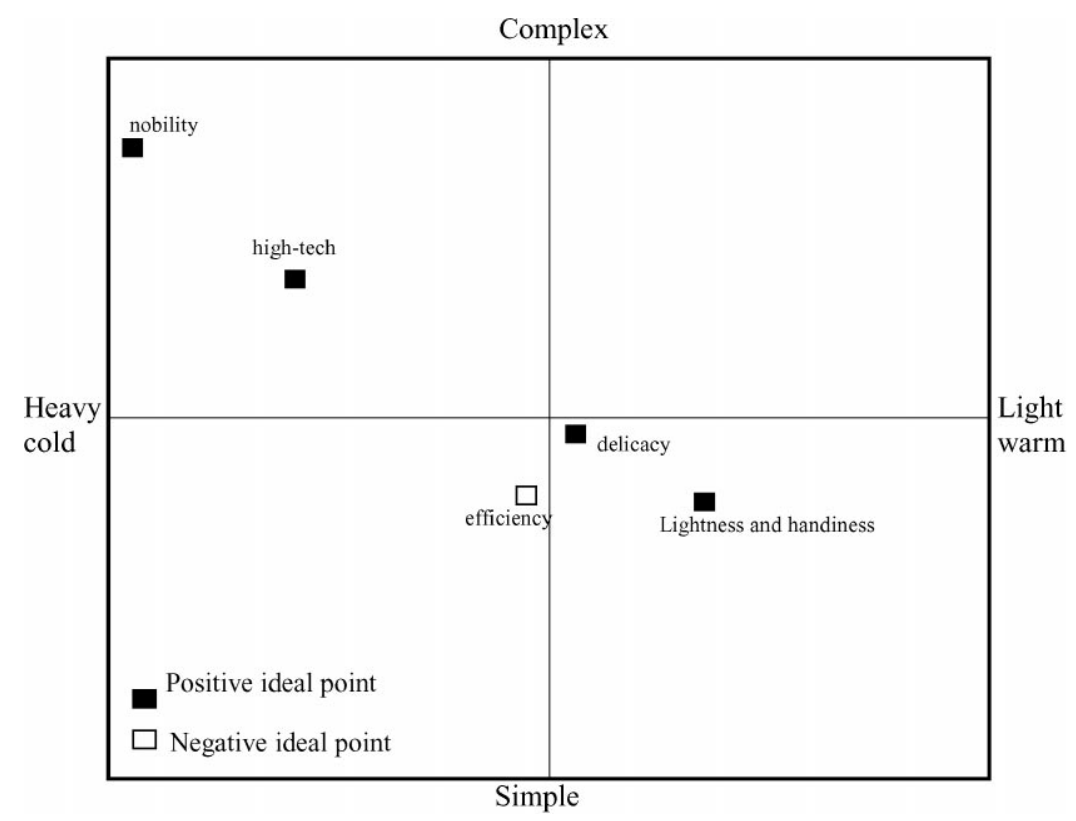

Fig. 5. The ideal points of the five most expected images in the perceptual space of micro-electronic products.

point of 'light and handy' image is located on the lower right of this map, products with somewhat light and simple forms will produce this kind of image. Finally, the negative ideal point of an 'efficient' image is located near the original point of this map too. Therefore, products with a distinct form, either light or heavy; either complex or simple, are more likely to evoke this image.

\subsection{The correspondence between expected images and form features}

During the first step of morphological analysis, several critical form features were identified by the first three experienced Industrial Designers. From the oral explanations on how subjects make their decisions in selecting the representative products for expected images, other critical form features were also extracted. Combining these two results, 35 critical form features (depicted as F1-F35 in Table 4), including form elements, form treatments, finishing treatments, material selections and color schemes have been summarized.
During the second step of morphological analysis, each of the 30 products was evaluated with each of these 35 form features by the second three experienced Industrial Designers. This resulted in a form attribute matrix of these 30 products. Then, for each expected image, we took the expressive power of the tested products as dependent variables and the elements of this form attribute matrix as independent variables to establish a set of multiple linear equations. A stepwise multiple linear regression analysis was then conducted for each set of equations. The result of the regression analysis for each expected image can be expressed as an equation and a table containing the coefficients of some critical form features. For example, Table 3 depicts the regression result of a 'high-tech' image. In this table, the significant form features (at the significant level of $0.1)$ were listed in the first column; whereas their corresponding coefficients $(b)$ were listed in the second column. Therefore, the correspondence between the 'high-tech' image and some significant form features can be expressed as the following 
Table 3

The regression coefficients of the significant form features to a 'high-tech' image (at the significant level of 0.1 )

\begin{tabular}{lrlrrr}
\hline \multirow{2}{*}{ Feature } & \multicolumn{2}{l}{ Unstandardized coefficients } & Beta & & Sig. \\
\cline { 2 - 3 } & \multicolumn{1}{c}{$B$} & $\mathrm{SE}$ & & & \\
\hline Constant & 4.26 & 1.74 & & 2.45 & 0.027 \\
F6 & -1.02 & 0.55 & -0.27 & -1.87 & 0.082 \\
F10 & 3.60 & 0.66 & 0.95 & 5.43 & 0.000 \\
F11 & -1.79 & 0.73 & -0.55 & -2.44 & 0.028 \\
F12 & 2.27 & 0.65 & 0.57 & 3.52 & 0.003 \\
F14 & 1.98 & 0.57 & 0.52 & 3.44 & 0.004 \\
F15 & -1.86 & 0.56 & -0.44 & -3.34 & 0.004 \\
F16 & -2.18 & 0.83 & -0.34 & -2.62 & 0.019 \\
F17 & 3.05 & 0.67 & 0.81 & 4.58 & 0.000 \\
F20 & 2.49 & 0.62 & 0.66 & 4.00 & 0.001 \\
F22 & -1.91 & 0.64 & -0.41 & -2.99 & 0.009 \\
F23 & -1.46 & 0.70 & -0.29 & -2.09 & 0.055 \\
F30 & 5.57 & 1.28 & 0.70 & 4.34 & 0.001 \\
F31 & -2.42 & 0.61 & -0.62 & -3.94 & 0.001 \\
F33 & -4.30 & 1.35 & -0.53 & -3.19 & 0.006 \\
\hline
\end{tabular}

equation $\left(R^{2}=0.83\right)$ :

Degree of 'high-tech' image

$$
\begin{aligned}
= & 4.26-1.02(\mathrm{~F} 6)+3.60(\mathrm{~F} 10)-1.78(\mathrm{~F} 11) \\
& +2.27(\mathrm{~F} 12)+1.97(\mathrm{~F} 14)-1.86(\mathrm{~F} 15) \\
& -2.18(\mathrm{~F} 16)+3.05(\mathrm{~F} 17) \\
& +2.49(\mathrm{~F} 20)-1.91(\mathrm{~F} 22) \\
& -1.46(\mathrm{~F} 23)+5.57(\mathrm{~F} 30) \\
& -2.42(\mathrm{~F} 31)-4.30(\mathrm{~F} 33) .
\end{aligned}
$$

According to this equation, the form features with positive coefficients should be adopted to enhance the degree of a 'high-tech' image; on the other hand, the corresponding form features with negative coefficients should be avoided to maintain the degree of a 'high-tech' image. The relative influence of these form features can be decided by comparing the absolute values of the normalized coefficients $(\beta)$, as shown in the forth column in Table 3. Thus, the form features that should be adopted to enhance the 'high-tech' image can be concluded as the following order of relative importance:

1. sound output holes arranged in a wave or radial pattern;
2. small but delicate components, such as buttons and LED indicators;

3. gold-plated knobs on black and polished operation panel;

4. parallel grooves with equal intervals spread in a fixed area;

5. plain shape without any decorative line or pattern;

6. with sudden drop connecting two parallel surfaces.

On the other hand, the form features that should be avoided to maintain the 'high-tech' image can be concluded as the following in order of relative importance:

1. color scheme with achromatic colors as principal colors;

2. color combination with low contrast or identical hue colors;

3. specially emphasized or exaggerated buttons or knobs;

4. form elements arranged in a gradual pattern, such as decrease or increase in size gradually;

5. highly polished or mirror surface finishing;

6. tiny holes arranged in a line or any distinctive shape pattern;

7. asymmetric balanced in form.

In the same way, the correspondences between each of the other four expected images and some significant form features can be derived from the regression analyses. Table 4 summarizes the regression results of the five expected images. In this table, a ' + ' mark at the intersection of a specific expected image and a form feature denotes that the form features have to be adopted to enhance the expected image. On the other hand, a '-' mark denotes the specific form feature that should be avoided to maintain the corresponding image.

\section{Conclusion}

Drawn from the above discussion several findings from this study can be summarized as the following. 
Table 4

The form features to be adopted or should be avoided for enhancing expected images

\begin{tabular}{|c|c|c|c|c|c|c|}
\hline & \multirow[t]{2}{*}{ Form elements or treatments } & \multicolumn{5}{|l|}{ Image } \\
\hline & & High-tech & Efficiency & $\begin{array}{l}\text { Lightness and } \\
\text { handiness }\end{array}$ & Nobility & Delicacy \\
\hline F1. & Organic shape & & & & & $-{ }^{\mathrm{a}}$ \\
\hline F2. & Small rounded corner & & & - & & - \\
\hline F3. & Large rounded corner & & & - & - & \\
\hline F4. & Stair shape & & & + & & - \\
\hline F5. & Symmetric balance & & & & & - \\
\hline F6. & Asymmetric balance & - & & & - & \\
\hline F7. & With dividing lines of equal interval & & - & - & + & + \\
\hline F8. & Condensed tiny holes in a fixed area & & - & - & + & - \\
\hline F9. & Convex curvature surface & & & & & + \\
\hline F10. & $\begin{array}{l}\text { Sound output holes arranged in a wave or radial } \\
\text { pattern }\end{array}$ & $+{ }^{b}$ & + & - & & - \\
\hline F11. & $\begin{array}{l}\text { Specially emphasized or exaggerated buttons or } \\
\text { knobs }\end{array}$ & - & & - & & - \\
\hline F12. & $\begin{array}{l}\text { Plain shape without any decorative line or } \\
\text { pattern }\end{array}$ & + & + & - & & + \\
\hline F13. & $\begin{array}{l}\text { With decorative lines or patterns of antique or folk } \\
\text { style }\end{array}$ & & & & & + \\
\hline F14. & With sudden drop connecting two parallel surfaces & + & & & + & \\
\hline F15. & $\begin{array}{l}\text { Form elements arranged in gradual pattern, such } \\
\text { as decrease or increase in size gradually }\end{array}$ & - & & - & & \\
\hline F16. & $\begin{array}{l}\text { Tiny holes arranged in a line or any distinctive } \\
\text { shape pattern }\end{array}$ & - & + & + & - & + \\
\hline F17. & $\begin{array}{l}\text { Small but delicate components, such as buttons } \\
\text { and LEDs }\end{array}$ & + & + & + & & \\
\hline F18. & With clear instruction icons or labels & & & & & + \\
\hline F19. & With exposed screws or inserted parts & & & + & & + \\
\hline F20. & $\begin{array}{l}\text { Parallel grooves of equal intervals spread in a } \\
\text { fixed area }\end{array}$ & + & + & - & & - \\
\hline F21. & Matted finishing & & & & & + \\
\hline F22. & Polished finishing & - & & & & \\
\hline F23. & Mirror surface finishing & - & & & + & + \\
\hline F24. & Sandblasted finishing & & & - & + & \\
\hline F25. & Granular surface finishing & & & & & - \\
\hline F26. & Using transparent material & & & & & \\
\hline F27. & Using wood with metal material & & & & & \\
\hline F28. & Emphasized wood texture & & & & - & \\
\hline F29. & $\begin{array}{l}\text { Identifying areas of different function by different } \\
\text { textures }\end{array}$ & & & + & & - \\
\hline F30. & $\begin{array}{l}\text { Gold-plated knobs on black and highly } \\
\text { polished operation panel }\end{array}$ & + & & - & + & \\
\hline F31. & Achromatic color scheme & - & & + & - & \\
\hline F32. & Color combination of colors of low saturation & & & + & & \\
\hline F33. & $\begin{array}{l}\text { Color combination of colors of low contrast } \\
\text { or identical hue }\end{array}$ & - & & + & & \\
\hline F34. & Contrasted color combination of different hues & & & & & \\
\hline F35. & $\begin{array}{l}\text { Identifying areas of different function by different } \\
\text { colors }\end{array}$ & & + & & - & \\
\hline
\end{tabular}

\footnotetext{
a - Should be avoided.
}

b + To be adopted. 
1. The five most expected images of microelectronic products are 'high-tech', 'efficiency', 'lightness and handiness', 'nobility' and 'delicacy'.

2. There is only a marginal difference in expected images of micro-electronic products amongst individuals of differing backgrounds.

3. The mental or psychological factors constituting these expected images can be classified into three factors: (i) emotion (ii) stability and (iii) simplicity.

4. The 'high-tech', 'noble' and 'delicate' images are supported by a sensational, rational, stable, but luxurious feeling. However, the 'high-tech' image is comparatively more rational and stable; whereas the 'noble' and 'delicate' images have relatively higher sensational feeling. The 'efficient' image is similar to the 'hightech' image minus its 'simple' and 'plain' feeling as opposed to a 'complex' and 'luxurious' feeling. Thus, the image corresponds to the 'sensational', 'rational', 'stable', 'simple', and 'plain' feeling. The 'light and handy' image is very close to the 'simple' and 'plain' feeling and is somewhat related to the 'frivolous' and 'emotional' feeling.

5. Individuals' perceptions in the differentiation of the form of micro-electronic products can be expressed as a perceptual map constituted by two factors of simplicity and lightness/warmth perceived in the product forms.

6. Products with complex and heavy geometric forms may look more 'high-tech'; whereas products with complex and heavy forms and special finishing treatments may tend to have a 'noble' image. Products with a moderate form and special finishing treatment may give a 'delicate' image; products with a 'light and handy' image are always small, light, thin and simple. The 'efficient' image is easier to find from products with a distinctive geometric form.

7. The correspondence between several form features and the five expected images has been found and summarized as a design recommendation table in Table 5. In this table, designers can find the advice as to whether a specific form feature should be adopted or should be avoided in order to enhance expected images.
These findings provide us with a systematic understanding about the expectations and perceptions of people regarding micro-electronic products. This cannot only be directly applied to the design of forms of micro-electronic products, but provide insights into correspondence between product form and the mental feeling users may experience and therefore may serve as the basis for further studies. In addition, the structure of the study may be extended to further investigate product form and image of other products.

Due to the limitation of resources, the products investigated in this study were not market-placed ones but visual representations of such products. This may have somewhat influenced the subjects' evaluations. The interactions among some form features may generate special influences on product images, however due to the complexity of this problem, they were not considered in this study. The design recommendations obtained from this study have not as yet been verified, thus providing a opportunity for further study.

\section{References}

Caplan, S., Faulkner, T., 1982. Disc camera configuration and user preference. The Third National Symposium on Human Factors and Industrial Design in Consumer Products, The Ohio State University, OH, pp. 267-275.

Chan, C.S., 1992. Exploring individual style in design. Environment and Planning B: Planning and Design 19, 503-523.

Chan, C.S., 1993. How an individual style is generated. Environment and Planning B: Planning and Design 20, 391-423.

Chan, C.S., 1994. Operational definition of style. Environment and Planning B: Planning and Design 21, 223-246.

Chuang, M.C., Shiau, K.A., 1998. A study of style recognition and the operation of products in which Ming-style chairs are used as examples. Environment and Planning B: Planning and Design 25, 837-848.

Chuang, M.C., Shih, W.M., 1996. The study of the corresponding relationship between forms and images of eyeglasses. Proceedings of the Fourth Pan Pacific Conference on Occupational Ergonomics, Taipei, pp. 472-475.

Espe, H., 1992. Symbolic qualities of watches. In: Vihma, S. (Ed.), Objects and Images: Studies in Design and Advertising. University of Industrial Arts, Helsinki, pp. 86-93.

Fischer, Y. (Ed.), 1989. Design Now, Prestel-Verlag, Munich. 
Green, P.E., Carmone, Jr., F.S., Smith, S.M., 1989. Multidimentional Scaling-Concepts and Applications. Allyn \& Bacon, Boston. 407pp.

Industrial Forum Design Hanover, 1996. IF Yearbook of Industrial Design. Stora Papyrus Deutschland $\mathrm{GmbH}$, Germany.

Industrial Forum Design Hanover, 1997. IF Yearbook of Industrial Design. Bangert Verlag, Munich.

Japanese Association of Industrial Design Promotion, 1997. GOODDESIGN 1996-1997. Maruzan Ltd., Tokyo.

Jindo, T., Hirasago, K., Nagamachi, M., 1995. Development of a design support system for office chairs using 3-D graphics. International Journal of Industrial Ergonomics 15 (1), 49-62.

Mastandrea, S., Zani, A., Giuliani, M.V., Bove, G., 1992. Meaning of industrial design object : from designers to users. Environment and Planning B: Planning and Design 19, 307-319.

Maurer, C., Overbeeke, C.J., Smets, G., 1992. The semantics of street furniture. In: Vihma, S. (Ed.), Objects and Images:
Studies in Design and Advertising. University of Industrial Arts, Helsinki, pp. 86-93.

Nagamachi, M., 1995. Kansei engineering: a new ergonomic consumer-oriented technology for product development. International Journal of Industrial Ergonomics 15 (1), 3-11.

Nakada, K., 1997. Kansei engineering research on the design of construction machinery. International Journal of Industrial Ergonomics 19 (2), 129-146.

Osgood, C.E., Suci, C.J., 1957. The Measurement of Meaning. University of Illinois Press, Urbana.

Pearlman, D., 1996. I.D.-42 ${ }^{\text {nd }}$ Annual Design Review, I.D. Magazine July/August 1996, New York.

Sokolov, J., 1990. Product Design 4. PBC International Inc., New York.

Takahaski, S., 1995. Aesthetic properties of pictorial perception. Psychological Review 102 (4), 671-683.

Viemeister, T., 1993. Product Design 6. PBC International Inc., New York. 